\title{
Intervención Conductual-Contextual para paciente con problema de fase de vida: ruptura de relación de pareja
}

\section{Behavioral-Contextual Intervention for a patient with a life phase problem: breakup romantic relationship}

\author{
Alcázar Zapata, Diana Lizeth ${ }^{1, *}$ y Hernández-Brussolo Ricardo ${ }^{1}$
}

\begin{abstract}
Resumen:
La terapia conductual-contextual, basada en el Modelo Integrativo de Regulación Emocional de Reyes y Tena (2016), es efectiva y eficiente en el abordaje del Problema de fase de la vida: ruptura en la relación de pareja, debido a que adapta las técnicas para mejorar la calidad de vida de pacientes con problemas afectivos y emocionales crónicos. Este trabajo presenta la intervención con un paciente masculino de 24 años, que concluyó una relación amorosa con quien fue su pareja durante cinco años. El participante experimentó tristeza, dolor, angustia y enojo, consumo de alcohol y cambios en el apetito. Se trabajó con una metodología de diseño de estudio de caso único, y una intervención terapeutica consistente en 15 sesiones, con el objetivo de lograr regulación emocional, habilidad de solucionar problemas, reducir manifestaciones físicas y fomentar consciencia plena. Los resultados muestran que la terapia con el Modelo Integrativo de Regulación Emocional logra una disminución de la sintomatología presente en el Problema de fase de la vida, por lo que se concluye la efectividad del enfoque Conductual-Contextual.
\end{abstract}

Palabras Clave: psicología, clínica, problema fase de vida, intervención conductual-contextual, salud mental.

\begin{abstract}
:
Behavioral-contextual therapy, based on the Integrative Model of Emotional Regulation of Reyes and Tena (2016), is effective and efficient in dealing with the problem of phase of life: breakup romantic relationship, because it adapts its techniques to improve the quality of life of patients with chronic affective and emotional problems. This work presents an intervention with a male patient 24 years old, who concluded a romantic relationship with whom was his partner for five years. He experienced sadness, pain, anguish and anger, alcohol consumption and changes in appetite. The methodology used is a unique case study design, and a therapeutic intervention consisting of 15 sessions, with the aim of achieving emotional regulation, ability to solve problems, reduce physical manifestations and promote full awareness. Results show that therapy with the Integrative Model of Emotional Regulation achieves a reduction of the symptomatology present in the Life phase problem, so that the effectiveness of the Behavioral-Contextual approach is concluded.
\end{abstract}

Keywords: psychology, clinical, life phase problem, behavioral-contextual intervention, mental health.

\footnotetext{
${ }^{1}$ Secretaría de Salud de Tamaulipas. Centro Integral de Salud Mental, Ciudad Victoria, Tamaulipas, México.

*Correspondencia: psic_alcazar@outlook.com
} 


\section{Relación de noviazgo}

Los seres humanos, durante los periodos de adolescencia y juventud, inician relaciones interpersonales significativas, como el noviazgo, que permite al individuo cubrir necesidades esenciales para su desarrollo personal y social, entre estas necesidades están la confianza, protección, y amor (Barajas, González, Cruz y Robles, 2012).

El noviazgo ocurre cuando dos personas están en una condición de novios, es decir, que sostiene relaciones amorosas con fines matrimoniales (Real Academia Española [REA], 2018).

González y Díaz-Loving (2015) definen que una pareja se crea cuando dos personas toman la decisión de unirse, y fundar un sistema nuevo, que mediante el ensayo y error, aprenden a convivir, y a su vez establecen un nivel de intimidad y acercamiento. Hernández, Hernández, Parra, Peña y Zamorano (2013) afirman que las relaciones de pareja adquieren un papel importante en el desarrollo socioemocional, ya que brindan un recurso de apoyo social que contribuye al bienestar psicosocial y al afrontamiento de situaciones estresantes.

No obstante, por tratarse de la interacción entre dos personas con diferentes características personales, experiencias, actitudes y creencias, surge la posibilidad de que surja un conflicto y su dinámica se vea fragmentada, llegando al punto en ocasiones de romper el vínculo amoroso, ya sea de manera temporal o permanente (Barajas, González, Cruz y Robles, 2012).

La teoría del apego de Bowlby (García, Guzmán-González, Sandoval, Vásquez y Villagrán, 2014), postula que los vínculos que se establecen en etapas tempranas de la vida, quedan registrados como representaciones denominadas modelos operati- vos internos de sí mismo, que influyen en la manera de enfrentar las relaciones afectivas adultas (Guzmán, Santelices y Trabucco, 2015). En el apego adulto, se despliegan dos dimensiones para afrontar vínculos de intimidad: la ansiedad o temor al abandono y la evitación de la cercanía e intimidad (Guzmán, Santelices, y Trabucco, 2015).

\section{Rasgos de personalidad evitativa}

Para la psicología y psiquiatría, la personalidad surge de una base genética, se va formando con las interacciones recíprocas de la persona con otros, mientras que los rasgos de personalidad son los patrones característicos de pensamientos, conductas y sentimientos (Sanjuán, Guillén y Pérez-García, 2018).

Las personas que padecen algún trastorno de personalidad siguen pautas fijas de percepción de la realidad y patrones rígidos de conducta en la sociedad. Generalmente, no muestran flexibilidad y destreza suficientes para adaptarse a situaciones cambiantes e inestables de la sociedad, y no son conscientes de que siguen modelos inadecuados de percepción, pensamiento y conducta (Haranburu, Esteve, Guerra, Balluerka, y Gorostiaga, 2011).

Entre los rasgos característicos de una persona evitativa están: la inhibición social, sentimientos de impotencia e hipersensibilidad hacia la evaluación negativa realizada por otras personas. Poseen baja autoestima y valoran de manera negativa sus facultades y logros, muestran temor al fracaso, la crítica y el rechazo. Sin embargo, algunas personas evitativas consiguen relaciones afectivas satisfactorias (Haranburu, Esteve, Guerra, Balluerka, y Gorostiaga, 2011).

\section{Regulación emocional}

Los procesos conductuales subyacen a la 
emoción y a la regulación emocional. La regulación emocional es el proceso a través del cual los individuos influyen en las emociones que experimentan y la forma en que las expresan, con ellos mismos y otras personas (Reyes y Tena, 2016).

La regulación emocional es cualquier intento realizado por una persona para influir en su experiencia y expresión emocional (Reyes, 2016). La regulación emocional tiene un amplio componente motivacional que funciona para encaminar y lograr las metas, así como la adaptación biológica y social del individuo. Para lograr la regulación se cuenta con este modelo procesual, que contiene cinco categorías: selección situacional, modificación situacional, manipulación de la atención, revaloración cognitiva y modulación de la respuesta (Reyes y Tena, 2016).

\section{Modelo Integrativo de Regulación Emocio- nal (MIRE)}

El Modelo Integrativo de Regulación Emocional es un tratamiento conductual de tercera generación, es decir, conductual-contextual, que tiene como premisa principal que la base de los problemas es la evitación experiencial. Este modelo lo inició en México Michel André Reyes Ortega, en 2010 (Reyes, 2015; Reyes, 2016).

El objetivo principal del modelo es la resolución de dificultades para la regulación emocional, para mejorar la calidad de vida de los pacientes con problemas afectivos y emocionales crónicos, desarrollar una actitud de aceptación ante el malestar psicológico, desarrollar habilidades para solucionar problemas, y las habilidades relacionadas con la construcción de relaciones sociales constructivas y duraderas (Reyes, 2015).

El tratamiento psicológico, de acuerdo al MIRE, de forma similar a otros modelos cognitivo-conductuales, tiene como pasos: validar la experiencia emocional del paciente, se identifican las estrategias improductivas para sustituirlas por las efectivas (ayuda a fortalecer las habilidades de regulación emocional). Posteriormente, se replantean las direcciones de vida del paciente con tácticas de clarificación de valores (fortalece las habilidades de la persona para cuidarse a sí misma y mantener una dirección valorada), después se revalúan los supuestos emocionales y personales, finalmente se le prepara al paciente para prevenir recaídas (Reyes, 2015).

El MIRE permite que el o la psicoterapeuta diseñe el tratamiento acorde al paciente que acude al servicio psicológico. Debido a los diferentes factores que componen a la persona, el psicólogo tiene el compromiso de realizar un estudio del caso respetando hasta los detalles que parezcan más sencillos como la edad, raza, religión, síntomas y motivos particulares del paciente a tratar, también respetando las características de su estilo como terapeuta.

La intervención con el MIRE se considera efectiva cuando las acciones de regulación se adecuan a las características de la persona que vive el problema; mientras que la regulación emocional se considera no efectiva cuando la persona no logra desarrollar expresión emocional, se mantiene con impulsividad o inhibición de la experiencia (Leahy, Tirch \& Napolitano, 2011; Reyes, 2016).

\section{Caracteristicas del individuo}

El paciente L. G., es un hombre de 24 años de edad, que acude a consulta por intención personal, refiriendo como principal motivo la ruptura amorosa con su novia, dos meses previos a llegar a consulta. Debido a la tristeza, dolor, angustia y enojo que sentía a causa de la separación, se inició en el consumo de al- 
cohol, además de tener cambios en el apetito, ya que disminuyó su ingesta de comida, y también modificó sus hábitos de sueño, pues dormía más por las tardes y no en las noches.

Menciona que su ex novia terminó la relación el día de su quinto aniversario. Relata que fue amable con él y que le entregó una carta explicándole sus razones para no continuar con la relación, entre lo que le decía era que lo quería mucho porque era buena persona, pero ya no lo amaba, que ahora ella necesitaba tiempo para saber qué era lo que quería de su vida, le pedía perdón y le externaba su amistad. A pesar de que él ya presentía que sucedería, no esperaba que se fuera a sentir tan mal: "ya llevaba varios meses sintiendo que algo no andaba bien, ya nos veíamos poco y ella no era tan cariñosa conmigo como antes...".

Su historia como pareja comenzó de manera cordial y romántica, se conocían desde la secundaria, pero fue durante la preparatoria que se hicieron amigos, platicaban mucho y se frecuentaban para salir, por lo que decidieron iniciar un noviazgo, y dejar la comunidad donde vivían para mudarse a la ciudad y estudiar la universidad, aunque estarían en carreras distintas. A ambos les rentaron sus papas un lugar dónde vivir: "ella estaba en un departamento y yo en una casa chica”. El participante cuenta que se veían con frecuencia durante la semana, y los fines de semana se iban juntos a su lugar de origen.

Cuando tenían tres años de noviazgo, decidieron cohabitar sin mencionárselo a sus familias, porque les habían dado cierta educación que no permitía vivir con la pareja antes de casarse. Compartieron casa durante ocho meses, hasta que él egresó de la universidad y regresó unos meses a su lugar de origen, fue en ese momento cuando su novia empezó a cambiar con él. Ella entró al servicio social y le decía que andaba siempre cansada, hablaban menos por celular, y se veían poco, sólo se reunían los fines de semana.

L. G. fue aceptado para trabajar como empleado de gobierno, por lo que se mudó a vivir solo a otra ciudad, esto le ocasonó tristeza, reducción del apetito, e inseguridad, pues tenía que relacionarse con personas que no conocía. Menciona que a partir de la mudanza, notó que la relación se volvió distante de forma física y emocional; dejó de llamarla, por temor a obtener una respuesta que confirmara la ruptura; el miedo que sentía a enfrentar el fin de la relación, prolongó la agonía que sentía, incluso dejó de responderle mensajes y evitar contestar las llamadas. Fue entonces cuando inició el consumo de alcohol, y dejó de comer de forma regular.

Relata que sus pensamientos se volvieron catastróficos, y se centraban en la ruptura de la relación, pero no se sentía seguro de afrontar el problema, por eso evitaba hablar con ella, situación que duró cinco meses. Finalmente, en su quinto aniversario, ella terminó la relación con L. G.

El objetivo de la intervención fue lograr regulación emocional eficaz, eficiente y efectiva en el participante, desarrollar sus habilidades para solucionar problemas, reducir malestares emocionales y físicos, y fomentar consciencia plena.

\section{Método}

Diseño de estudio de caso único. Se utilizó un diseño de una sola persona $(N=1)$ del tipo AB (Marco y Chóliz, 2014), abordándose como intervención psicoterapéutica, evaluada antes y después del tratamiento.

El análisis de un caso permite investigar los contextos discursivos en los que acontece (Grinsvall y Lora, 2012). Es una forma de transmitir ese conocimiento y cómo puede 
alcanzar su materialización escrita. El estudio de caso, es el acto de pasar a lo público (Sánchez, 2016).

\section{Participante}

Hombre de 24 años de edad, que atraviesa por ruptura amorosa.

\section{Escenario}

Las pruebas se aplicaron por parte de ambos psicoterapeutas, en uno de los consultorios del Centro Integral de Salud Mental (CISAME), Victoria, Tamaulipas, México. Perteneciente a la Secretaría de Salud de Tamaulipas, que contó con los requerimientos para que el paciente se sintiera cómodo. El paciente fue informado de los objetivos de la investigación al momento de la evaluación, e intervención, y firmó el consentimiento informado.

\section{Instrumentos de medición}

Se aplicaron seis instrumentos para evaluar al participante:

Mini examen del estado mental de Folstein (Valdes, González, y Salisu, 2017). Se trata de un test que mide el estado mental y detecta trastornos cognitivos. En cuanto a su fiabilidad, su consistencia interna es de entre 0.82 y 0.84 .

Entrevista conductual para adultos de Fernández-Ballesteros (Rodríguez, 2013). Se enfoca en recabar información sobre aspectos potencialmente relevantes para el diagnóstico clínico.

Evaluación de Medida de síntomas transversales de nivel 1 del DSM-5 autoevaluada: adulto (American Psychiatric Association [APA], 2013). Valora los ragos de personalidad patológicos de forma inicial, para la toma de decisión del diagnóstico y tratamiento clínico.
Cuestionario de Evaluación Internacional de Desordenes de Personalidad (Internacional Personality Disorder Examination, [I.P.D.E.]), (Esbec y Echeburúa, 2014). Explora conductas y rasgos relevantes para hacer el diagnóstico clínico psicológico. La concordancia se ha estudiado en 11 países de América del Norte, Europa, Africa y Asia, donde se ha concluido que la fiabilidad y estabilidad del IPDE para el diagnóstico de psicosis, trastornos de ansiedad, del humor, y por abuso de sustancias (Organización Mundial de la Salud, 1996).

Escala de Funcionamiento Psicológico Positivo (Merino, Privado, y Gracia, 2015). Mide recursos psicológicos como propósito de vida, autonomía, resiliencia, autoestima, creatividad, optimismo, curiosidad, humor, dominio del entorno y vitalidad. Con validez convergente superior a .70, correlacionado on otras escalas como la escala de bienestar psicológico (.76), con la escala de afecto positivo del PANAS(.74). La escala completa tiene una fiabilidad de .91, obtenido con un alfa de Cronbach.

Cuestionario VIA de Fortalezas Personales (Peterson \& Seligman, 2004). Aplicable a personas mayores de 18 años, y que mide 24 fortalezas. Sus escalas tienen una fiabilidad satisfactoria $(\alpha>$.70).

\section{Procedimiento}

Para llevar a cabo el tratamiento del paciente L.G. se seleccionó el Modelo Integrativo de la Regulación Emocional, que se agrupa dentro de las Terapias ConductualesContextuales (Reyes, 2015).

El modelo conductual ha tenido cambios substanciales, debido a que en sus inicios se caracterizaba por ser un modelo radicalmente externalista, en el cual la conducta era sólo la función de un estímulo, sin embar- 
go, gracias a las modificaciones, se reformó como un modelo interactivo en el que la conducta, las variables de la persona y el ambiente interaccionan solidariamente (FernándezBallesteros, 2013). Es por esta razón, que el modelo conductual es utilizado, desde la formulación actual, donde $\mathrm{C}=\mathrm{fP} \times \mathrm{A}$, para comprender el entorno y las conductas del paciente.

De acuerdo al MIRE, se obtiene como beneficio principal para el paciente: cambios en su comportamiento, considerando la parte emocional, debido a que las personas utilizan las emociones como normas heurísticas que manejan su capacidad para la toma de decisiones y la capacidad de resolver problemas (Reyes y Tena, 2016).

Entre las técnicas seleccionadas están: psicoeducación, activación conductual, entrenamiento en identificación de valores y comunicación asertiva, entrenamiento en regulación emocional, resolución de conflictos $\mathrm{y}$ prevención de recaídas, que permiten elevar el nivel de bienestar del paciente, fomentar su inteligencia emocional, aumentar su nivel de autoestima, mejorar sus habilidades para relacionarse con otras personas y detener las conductas problema.

La intervención constó de 15 sesiones: tres de evaluación, y nueve de intervención, y tres de seguimiento, de 60 minutos cada una, llevadas a cabo en el Centro Integral de Salud Mental (CISAME) Victoria, que pertinence a la Secretaría de Salud de Tamaulipas.

Se acordaron con el paciente los objetivos terapéuticos de mejorar la habilidad de solucionar problemas, mejorar la habilidad de comunicación asertiva, incrementar la regulación emocional, reducir manifestaciones físicas y niveles de irritabilidad, angustia, tristeza, dolor y enojo, reducir la evitación social, y fomentar habilidades de consciencia plena, como se muestra en la tabla 1.

\section{Resultados}

\section{Resultados de la evaluación}

Mini examen del estado mental de Folstein (Valdes, González, y Salisu, 2017), que indicó que el participante se encuentra orientado en persona, tiempo y espacio. La Entrevista conductual para adultos de FernándezBallesteros (Rodríguez, 2013), mostró que el participante presentaba tristeza, ira, dolor y angustia, además de problemas de sueño y de la ingesta. Con este instrumento, se desarrolló un genograma, mapa clínico de patogénesis de Nezu, Nezu y Lombardo (Caro, 2017), (que permite identificar la relación de las variables antecedentes, organísmicas, y de respuesta a corto y largo plazo), el mapa clínico de fortalezas (para ubicar las áreas positivas del sujeto), y el análisis funcional (con el que se verifica el nivel de funcionalidad del participante).

En la evaluación de Medida de síntomas transversales de nivel 1 del DSM-5 autoevaluada: adulto (APA, 2013), obtuvo una puntución de 2 , en los dominios de mania y personalidad, además de 1 en ira, ansiedad, problemas del sueño, y disociación. Sin embargo la puntuación 2 pertenece a un nivel leve, por lo que se consideró realizar una exploración más detallada para esclarecer los niveles de puntuación, utilizando el Cuestionario de Evaluación Internacional Personality Disorder Examination (I.P.D.E.), (Esbec y Echeburúa, 2014) en la que obtuvo una puntuación significativa (4) para el área de evitación, lo cual concuerda con lo comentado en la entrevista conductual, refiriendo tener problemas para relacionarse con otras personas. 
Tabla 1.

Tratamiento Conductual-Contextual para paciente con problema de la fase de vida: ruptura en la relación de pareja.

\begin{tabular}{|c|c|c|c|}
\hline Sesión & Objetivos & Técnicas & $\begin{array}{c}\text { Tareas entre sesio- } \\
\text { nes }\end{array}$ \\
\hline 1 & Evaluación del participante & $\begin{array}{l}\text { Entrevista conductual para adultos de } \\
\text { Fernández- Ballesteros (Rodríguez, } \\
\text { 2013). } \\
\text { Consentimiento informado (elaboración } \\
\text { propia). }\end{array}$ & $\begin{array}{l}\text { Realizar un diario: } \\
\text { colocar a manera de } \\
\text { lista tres situaciones } \\
\text { positivas que vivió } \\
\text { durante el día. }\end{array}$ \\
\hline
\end{tabular}

2 y 3 Evaluación del participante

\section{Intervención:}

Dar a conocer al paciente los resultados de las evaluaciones.

Establecer los objetivos terapéuticos y el contrato terapéutico.

Conocer la función de las emociones.

Conocer las estrategias de regulación emocional inefectivas.

Reducir manifestaciones físicas y niveles de irritabilidad, angustia, tristeza, dolor y enojo.Mejorar el estado de ánimo.

Proporcionar estrategias para cuestionar creencias negativas y estados emocionales improductivos.
Mini examen del estado mental de Folstein (Valdes, González y Salisu, 2017). Medidas de síntomas transversales de nivel 1 del DSM -5 autoevaluadas: adulto (American Psychiatric Association, 2013)

Cuestionario de Evaluación Internacional Personality Disorder Examination (I.P.D.E.), (Esbec y Echeburúa, 2014).

Escala de Funcionamiento Psicológico Positivo (Merino, Privado, y Gracia, 2015).

Cuestionario VIA de Fortalezas Personales (Peterson, \& Seligman, 2004).

Devolución de resultados de los test aplicados.

Mapa clínico de patogénesis, mapa de fortalezas, mapa de alcance de metas, y análisis funcional (Caro, 2017).

Revisión de video sobre emociones (Ekman y

Oster, 1979), realizado por Centro Integral de Salud Mental de Victoria, de la Secretaría de Salud de Tamaulipas [CISAME] (2017).

Activación conductual.

Identificación de valores.

Explicación de la tarea para casa: Cuadro de acciones de activación (Reyes y Tena, 2016).
Continuar con el diario: colocar a manera de lista tres cosas o situaciones positivas que vivió durante el día.

Continuar con el diario.

Continuar con el diario.

Registro de estrategias de regulación emocional improductivas.

Continuar con el diario. 
Tabla 1.

Tratamiento Conductual-Contextual para paciente con problema de la fase de vida: ruptura en la relación de pareja (continuación).

\begin{tabular}{ll}
\hline 7 & $\begin{array}{l}\text { Favorecer el autocontrol y la iden- } \\
\text { tificación de emociones. } \\
\text { Incrementar la regulación emo- } \\
\text { cional. }\end{array}$ \\
8 & \\
& \\
& Favorecer la autoestima. \\
& Reducir la evitación social. \\
& haborcionar conocimiento sobre \\
& tiva. \\
& Fomentar e incrementar las habili- \\
dades de conciencia plena. & Lograr la relajar del participante.
\end{tabular}
Destrezas de regulación emocional efec-
tivas (Reyes y Tena, 2016).

Recomendación de cortometraje: validation (Kuenne, 2011).

Biblioterapia. Lectura del tema: La práctica de la inteligencia emocional (Goleman, 1998).

Técnica de relajación: respiración diafragmática (Mendoza, 2010).

Aceptación radical.

Comunicación asertiva.

Biblioterapia: La práctica de la inteligencia emocional (Goleman, 1998).

Psicoeducación sobre Mindfulness. Ejercicio de práctica de consciencia plena.

Biblioterapia recomendada: "La paz está en tu interior (Nhat, 2012).

Técnica de relajación (Mendoza, 2010).

Psicoeducación: Solución de problemas. Práctica de habilidad para solucionar problemas: Autoafirmación (Mahoney, 1991),

Disminuir la tendencia a evitar conflictos y la resolución impulsiva de los problemas.

Mejorar la habilidad de solucionar los problemas.

11
Realizar la evaluación post- tratamiento.
Lectura: "El radar social".

"Las artes de la influen-

cia" (Goleman, 1998).

Ejercicio de práctica de consciencia plena.

"La paz está en tu interior (Nhat, 2012).

Seguimiento de los ejercicios de práctica de consciencia plena.
Medidas de síntomas transversales de nivel 1 del DSM -5 autoevaluadas: adulto (American Psychiatric Association, 2013).

Cuestionario de Evaluación Internacional Personality Disorder Examination (I.P.D.E.), (Esbec y Echeburúa, 2014).

Escala de Funcionamiento Psicológico Positivo (Merino, Privado, y Gracia, 2015).

Cuestionario VIA de Fortalezas Personales (Peterson \& Seligman, 2004). 
Tabla 1.

Tratamiento Conductual-Contextual para paciente con problema de la fase de vida: ruptura en la relación de pareja (continuación).

$12 \begin{aligned} & \text { Concientizar acerca de los cam- } \\ & \text { bios positivos obtenidos de la tera- } \\ & \text { pia. } \\ & \text { Reconocer instancias de riesgo y } \\ & \text { realizar la planeación de conduc- } \\ & \text { tas funcionales alternativas para } \\ & \text { prevenir las recaídas. } \\ & \text { Seguimiento: } \\ & \text { Mantener los resultados favora- } \\ & \text { bles y afrontar los problemas ac- } \\ & \text { tuales por medio de las técnicas } \\ & \text { aprendidas del entrenamiento en } \\ & \text { regulación emocional. }\end{aligned}$

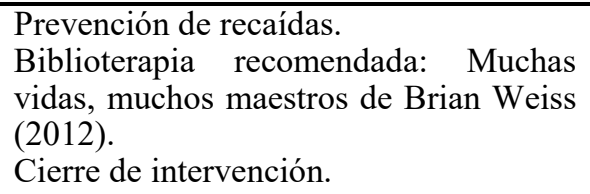

Seguimiento 1: a los dos meses de concluir el tratamiento.

Diario con la automonitorización sobre el malestar y los valores.

14 Mantener los resultados favorables y afrontar los problemas actuales por medio del entrenamiento en regulación emocional.
Seguimiento 2: A los dos meses.

Entrevista

Técnicas en regulación emocional: solución de problemas (Reyes y Tena, 2016).

Seguimiento 3: A los 6 meses.

Entrevista

Psicoeducación

Técnicas en regulación emocional: $\mathrm{Re}-$ fuerzo positivo (Reyes y Tena 2016).
Registro de soluciones en base a la regulación emocional.

Practicar lo aprendido durante la terapia y reforzar.

\begin{abstract}
Mantener los resultados favorables y afrontar los problemas acto en regulación emocional.
\end{abstract}

Fuente: Elaboración propia del Plan de Tratamiento, que constó de 15 sesiones: tres de evaluación, nueve de intervención, y tres de seguimiento, de 60 minutos cada una, llevadas a cabo en el Centro Integral de Salud Mental (CISAME) Victoria, que pertinence a la Secretaría de Salud de Tamaulipas.

De acuerdo a los resultados de la Escala de Funcionamiento Psicológico Positivo (Merino, Privado, y Gracia, 2015), el paciente obtuvo puntuaciones más altas en las áreas de autonomía, humor y curiosidad. Referente al Cuestionario VIA de Fortalezas Personales (Peterson \& Seligman, 2004) tuvo como fortalezas principales la amabilidad y generosidad, equidad y justicia, perdón y misericordia, posee la capacidad para amar y ser amado, honestidad y autenticidad. Esto, probablemente le otorgue las habilidades necesarias para salir de los problemas expuestos en la entrevista conductual, así como para conti- nuar en el proceso del tratamiento psicológico.

\section{Diagnóstico}

Con base en el Manual Diagnóstico y Estadístico de los Trastornos Mentales, en su quinta edición (APA, 2013) se diagnostica Z60.0 [V62.89] Problema de fase de la vida: ruptura en la relación de pareja.

Problema de fase de la vida se utiliza en el individuo cuyo problema no deriva de un trastorno mental sino de un cambio de vida, como el matrimonio, el divorcio, un empleo nuevo, un problema por nido vacío o el 
retiro. Además, se encontró que el participante presenta 301.82 (F60.6) Rasgos de personalidad evitativa.

De acuerdo con los lineamientos del Manual de Clasificación Estadística Internacional de Enfermedades y Problemas Relacionados con la Salud, Décima Revisión (Organización Panamericana de la Salud, 1995), el diagnóstico médico es: Sin trastornos psiquiátrico.

\section{Resultados de la intervención}

Las gráficas comparativas (Figuras 1, 2 y 3) que se presentan a continuación, exponen los resultados pre y post-tratamiento, con el paciente L. G. de 24 años de edad. Se indica mejoría en todos los aspectos evaluados.

En la evaluación de las Medidas de síntomas transversales de nivel 1 del DSM -5 autoevaluadas: adulto (APA, 2013), se puede observar que al inicio, obtuvo una puntución de 2 , en manía y personalidad, y posterior a la evaluación, fue de 1 . Además los valores de 1 que obtuvo en ira, y problemas del sueño desaparecieron, como se aprecia en la Figura 1.

En la Escala de Funcionamiento Psicológico Positivo (Merino, Privado, y Gracia, 2015), las puntuaciones más altas que obtuvo pre tratamiento fueron: las áreas de autonomía, humor, y curiosidad, y posterior al tratamiento se observa (Figura 2) que autonomía y humor se mantienen, mientras que aumentaron puntuación las áreas de autoestima, resiliencia, dominio del entorno, vitalidad, propósito vital y disfrute.

En el Cuestionario de Evaluación Internacional Personality Disorder Examination (I.P.D.E.), (Esbec y Echeburúa, 2014), resultó con una puntuación significativa (4) para el área de evitación, previo a la intervención;

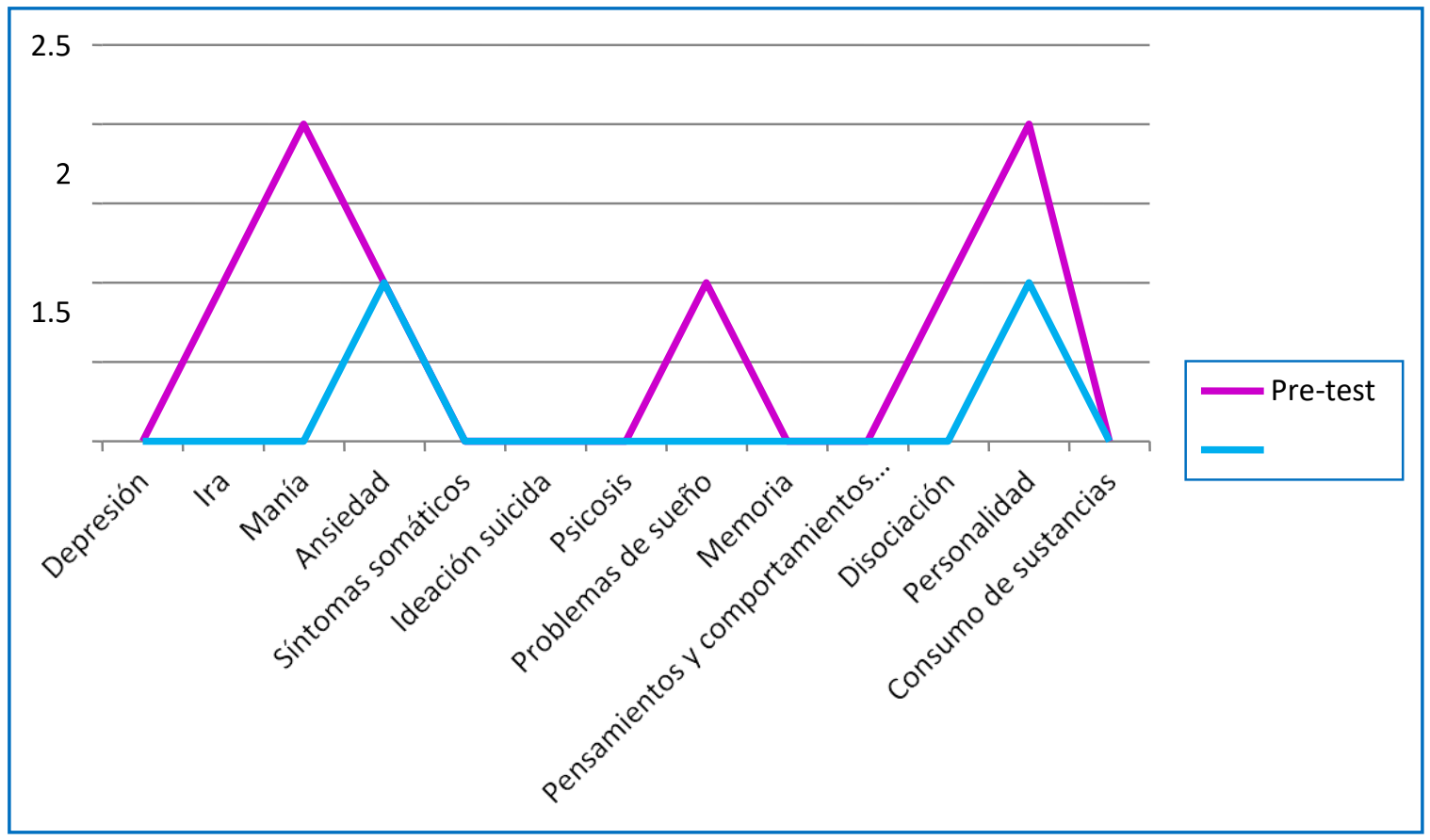

Figura 1. Resultados evaluación Medida de Síntomas Transversales de Nivel 1 del DSM-5 Autoevaluada: Adulto. 


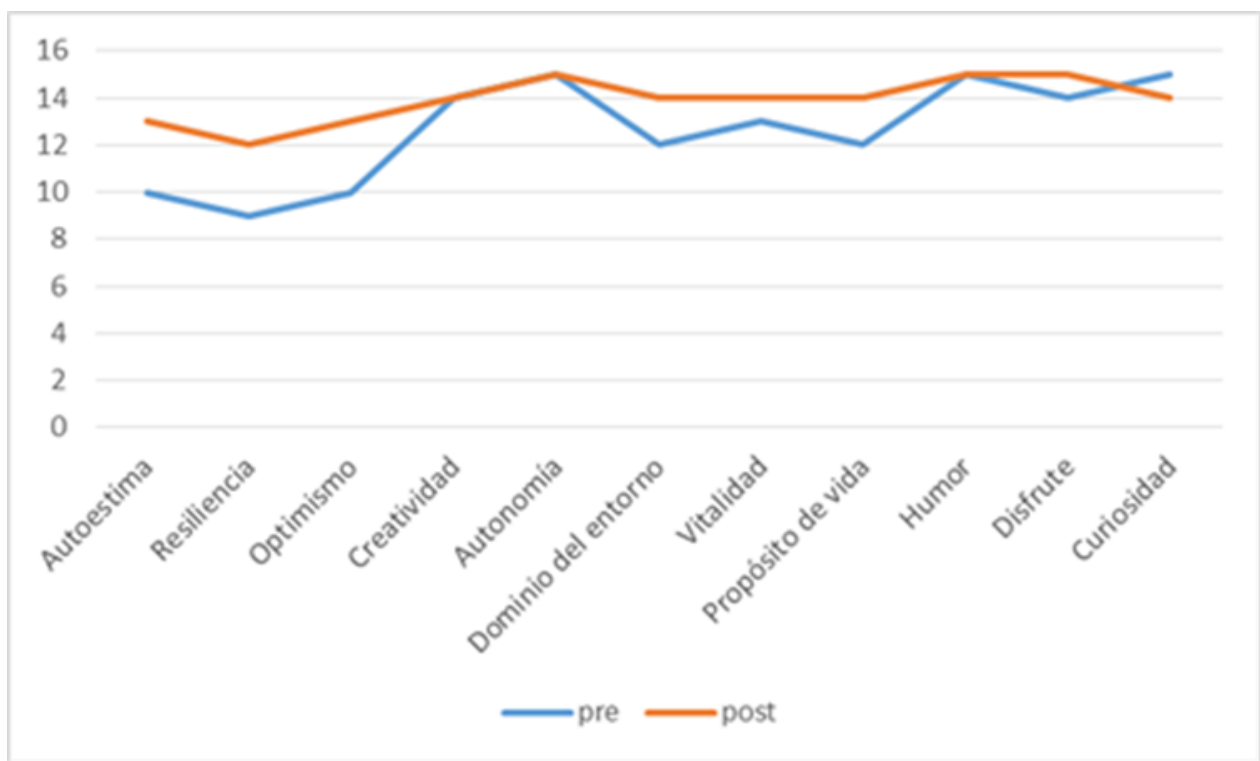

Figura 2. Resultados en Escala de Funcionamiento Psicológico Positivo (Merino, Privado, y Gracia, 2015).

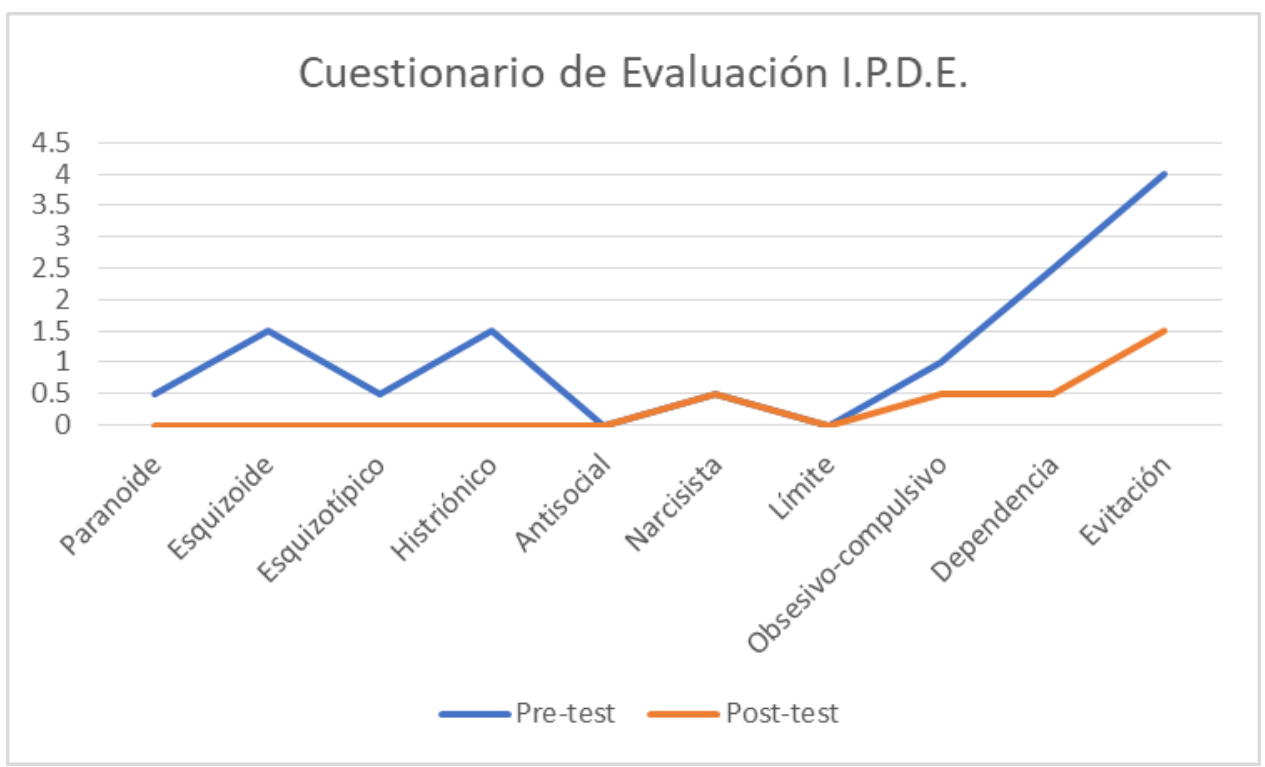

Figura 3. Evaluación pre y postratamiento con el Cuestionario de Evaluación Internacional Personality Disorder Examination (I.P.D.E.),(Esbec y Echeburúa, 2014) 


\section{Discusión y conclusiones}

El Modelo Integrativo de Regulación Emocional se desarrolla en México a partir del año 2010 (Reyes, 2013), como un tratamiento Conductual-contextual de tercera generación, que usa estrategias analítico-funcionales que se concentran en el paciente, su conducta, y aceptación del problema, para mejorar su salud mental (Reyes, 2016).

Este modelo de regulación emocional, propone que desde el área emocional, se logran cambios significativos en el comportamiento del paciente, su capacidad para tomar decisiones y resolver problemas (Reyes y Tena, 2016).

Las estrategias de atención psicológica como aceptación y mindfulness están tomadas de las terapias cognitivo-conductuales contextuales, que permiten una mejora significativa en la vida del paciente, sobre todo en el aspecto emocional y conductual (Padilla, 2013).

En el presente trabajo, se aplicaron pruebas al paciente, en dos momentos clave: al inicio y al final del tratamiento psicológico. De acuerdo a los valores obtenidos en las pruebas, se concluye que la Terapia Conductual- Contextual, desde el Modelo Integrativo de Regulación Emocional, demostró ser eficaz, eficiente y efectiva en esta intervención, para ayudar al participante con problemas de fase de la vida.

Se cumplieron los objetivos terapéuticos de mejorar la habilidad de solucionar problemas, mejorar la habilidad de comunicación asertiva, incrementar la regulación emocional, reducir manifestaciones físicas y niveles de irritabilidad, angustia, tristeza, dolor y enojo, reducir la evitación social, y fomentar habilidades de consciencia plena.
La relevancia de este tratamiento, se justifica en que al comprobar su eficacia, eficiencia y efectividad, puede ampliarse para aplicarse a otros pacientes con problemas emocionales, como el presentado en esta investigación.

Se encuentran como limitantes, la cantidad reducida de participantes, y problemáticas de salud mental abordadas. Se requiere incentivar a otros investigadores para continuar la aplicación del MIRE y ampliar los alcances terapéuticos del mismo, tanto en México, como otros países.

\section{Referencias}

American Psychiatric Association. (2013). Diagnostic and statistical manual of mental disorders (5th ed.) (Manual Diagnóstico y Estadístico de los Trastornos Mentales). Washington, DC: Author.

Barajas, M., González, C., Cruz, C., y Robles, R. (2012). El significado psicológico de una ruptura de pareja significativa en jóvenes universitarios. Psicología Iberoamericana, 20(2), 26-32. Recuperado de http://www.redalyc.org/ articulo.oa? id=133928816004

Centro Integral de Salud Mental Victoria (28 de febrero de 2017). Reconocimiento de emociones. Recuperado de https://www.facebook.com/ CISAMEVictoria/videos/1074478415992048/

Caro, C. (2017). Evaluación del modelo de formulación de caso de la terapia focalizada en la emoción (Tesis de Doctorado). Universidad Pontificia Comillas ICAI-ICADE, Madrid, España.

Esbec, E. y Echeburúa, E. (2014). La evaluación de los trastornos de la personalidad según el DSM-5: Recursos y limitaciones. Terapia psicológica, 32(3), 255-264. doi:10.4067/S071848082014000300008

Fernández-Ballesteros, R. (2013). Evaluación psicológica Conceptos, métodos y estudio de casos. Madrid, España: Ediciones Pirámide.

García, B., Guzmán-González, S., Sandoval, B., Vásquez, N. y Villagrán, C. (2014). Violencia psicológica en el noviazgo en estudiantes universitarios chilenos: diferencias en el apego y la empatía diádica. Revista Interamericana de Psico- 
logia/Interamerican Journal of Psychology, 48 (2), 338-346. Recuperado de https:// journal.sipsych.org/index.php/IJP/article/ view/272/pdf_47

Goleman. D. (1998). La práctica de la inteligencia emocional. Barcelona, España: Kairós.

González, I. y Díaz-Loving, R. (2015). Desarrollo y análisis psicométrico del Inventario de Indefensión Aprendida en la Pareja. Psicología Iberoamericana, 23 (1), 58-65. Recuperado de http:// www.redalyc.org/pdf/1339/133944230007.pdf

Grinsvall, S. y Lora Fuentes, M. E. (2012). La experiencia del exilio político y sus consecuencias en la subjetividad. Revista de psicología Ajayu, 10(2), 172-185. Recuperado de http:// www.ucb.edu.bo/publicaciones/Ajayu/v10n2/ v10n2a04.html

Guzmán, M., Santelices, M. y Trabucco, C. (2015). Apego y Perdón en el Contexto de las Relaciones de Pareja. Revista terapia psicológica, 33 (1), 35-45. Recuperado de http://teps.cl/ index.php/teps/article/view/25/27

Haranburu, M., Esteve, J., Guerra, J., Balluerka, N. y Gorostiaga, A. (Febrero-Marzo de 2011). Trastorno de personalidad evitadora: etiología y tratamiento. $12^{\circ}$ Congreso Virtual de Psiquiatría. Interpsiquis. Recuperado de: https:// www.evitacion.com/images/Trastorno-de\% 20personalidad-evitadora-etiologia-ytratamiento.pdf

Hernández, G., Hernández M., Parra, V., Peña, F. y Zamorano, B. (2013). Violencia en el noviazgo en una muestra de jóvenes mexicanos. Revista Costarricense de Psicología, 32(1), 27-40. Recuperado de http://www.rcps-cr.org/ openjournal/index.php/RCPs/article/view/17

Kuenne, K. [LucioA PerezV] (2011, enero 16). Cortometraje: Validation [Archivo de video]. Recuperado de https://www.youtube.com/watch? $\mathrm{v}=7$ ar TS $12 \mathrm{mP} 4$

Leahy, R.L., Tirch, D. \& Napolitano, L.A. (2011). Emotion regulation in psychotherapy: A practitioner's guide. New York, United States of America: The Guilford Press.

Mahoney, M., (1991). Human Change processes. Nueva York, United States of America: Basic Books.

Marco, C. y Chóliz, M. (2014). Tratamiento cognitivoconductual de la adicción a videojuegos de rol online: fundamentos de propuesta de tratamiento y estudio de caso. Anales de psicología, 30(1), 46-55. doi: 10.6018/ analesps.30.1.150851

Mendoza, B. (2010). Manual del enojo. Tratamiento Cognitivo-Conductual. México, D.F., México: Manual Moderno.

Merino M., Privado, J. y Gracia, Z. (2015). Validación mexicana de la Escala de Funcionamiento Psicológico Positivo. Perspectivas en torno al estudio del bienestar y su medida. Salud Mental, 38 (2) 109-115. Recuperado de: http:// www.scielo.org.mx/scielo.php? script $=$ sci_arttext\&pid $=\mathrm{S} 0185$ 33252015000200005

Nhat, T. (2012). La paz está en tu interior. Barcelona, España: Oniro.

Organización Mundial de la Salud. (1996). Examen Internacional de los Trastornos de la Personalidad. Modulo C.I.E. 10. Madrid: Meditor.

Organización Panamericana de la Salud. (1995). Clasificación estadística internacional de enfermedades y problemas relacionados con la salud10a. revisión. Washington, D.C.: OPS.

Padilla, D. (2013). Análisis retrospectivo de un protocolo asistencial cognitivo-conductual contextual grupal breve en adolescentes atendidos en un centro de salud mental público (Tesis doctoral). Universidad Autónoma de Madrid. Departamento de Psiquiatría, España.

Real Academia Española. (2018). Definición de noviazgo. España: Real Academia Española Recuperado de http://dle.rae.es/?id=QflTtlk

Reyes, M. A. (2015). Regulación emocional en usuarios con ansiedad generalizada. Reporte de la efectividad del modelo integrativo de regulación emocional grupal (MIRE-G). (Tesis de doctorado), Universidad Iberoamericana, Ciudad de México, México. Recuperada de: http:// www.bib.uia.mx/tesis/pdf/016015/016015.pdf

Reyes, M. A. (2016). Impactos del modelo integrativo de regulación emocional grupal en usuarios con trastorno de ansiedad generalizada. Enseñanza e Investigación en Psicología, 21 (1), 9-20. Recuperado de http:/www.redalyc.org/ pdf/292/29248180002.pdf

Reyes, M. A. y Tena, E. (2016) Regulación emocional en la práctica clínica: una guía para terapeutas. México: Manual Moderno.

Reyes, M.A. (2013). Características de un modelo clínico transdiagnóstico de regulación emocional. Psicología Iberoamericana, 21(2), 7-18. Recuperado de http://revistas.ibero.mx/psicologia/ articulo_detalle.php?

page- 
Num_paginas $=0 \&$ totalRows_paginas $=12 \&$ id $\_v$ olumen $=9 \&$ id_articulo $=99 \&$ pagina $=1$

Rodríguez, J. (2013). Guía de entrevista para el análisis funcional de conductas en personas transexuales. Papeles del Psicólogo, 34(2), 150-154. Recuperado de http://www.papelesdelpsicologo.es/ pdf/2238.pdf

Sánchez, J. (2016). Estudio de caso: una manera de investigar en psicoanálisis. Revista de psicología Ajayu, 14(1), 7-22. Recuperado de http:// www.ucb.edu.bo/publicaciones/Ajayu/v14n1/ v14n1a02.html

Sanjuán, P., Guillén, D. y Pérez-García, A. (2018). Rasgos de personalidad y recursos psicológicos como predictores del bienestar emocional en adolescentes con y sin formación en escuelas de tauromaquia. Revista de Psicopatología y Psicología Clínica, 23(1), 1-12. doi: 10.5944/ rppc.vol.23.num.1.2018.18494

Peterson, C., \& Seligman, M. E. P. (2004). Character strengths and virtues: A classification and handbook. Washington, DC: American Psychological Association/New York: Oxford University.

Valdes, M., González, J., y Salisu, M. (2017). Prevalencia de depresión y factores de riesgo asociados a deterioro cognitivo en adultos mayores. Revista Cubana De Medicina General Integral, 33(4), 1-15. Recuperado de http:// www.revmgi.sld.cu/index.php/mgi/article/ view/296/158

Weiss, B. (2012). Muchas vidas, muchos maestros. Barcelona, España: Zeta. 